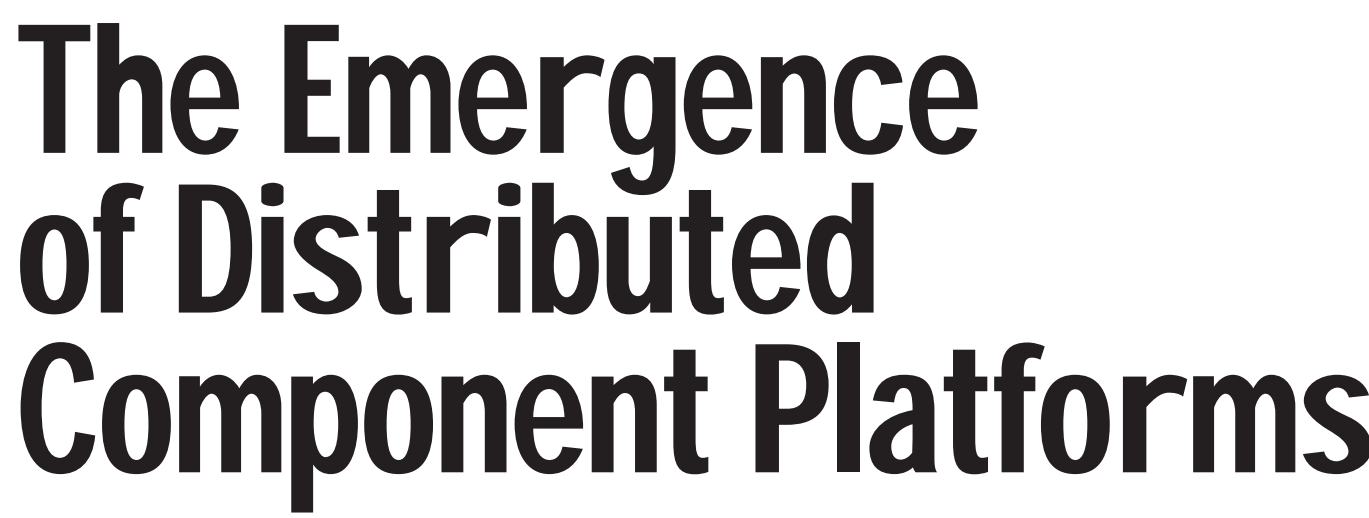

\begin{abstract}
Distributed component platforms isolate much of the conceptual and technical complexity involved in constructing component-based applications. The authors examine the concepts underlying DCPs, the two market leaders-Microsoft's DCOM and Sun's J avaBeans-and emerging Internet and OMG standards.
\end{abstract}

D avid Krieger
Richard M.
Adler
Computer
Sciences
Corp.

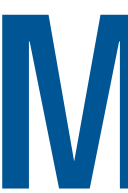

uch has changed in the world of component software since we last surveyed emerging standards three years ago. ${ }^{1}$ In that time, several vendors and consortia have independently developed standards that define the basic mechanics for building and interconnecting software components. Sun's J avaBeans has emerged as the leading rival to M icrosoft's DCOM (Distributed Component O bject $\mathrm{M}$ odel), supplanting the $O$ penD oc standard from the now defunct Component Integration Laboratories. Component software is moving from its original focus on desktop-bound compound documents to enterprise applications that include distributed server components.

The backers of competing standards are racing to capture market leadership by delivering the tangible benefits of component standards via distributed component platforms-integrated development and runtime environments that isolate much of the conceptual and technical complexity involved in building component-based applications. W ith DCPs, businesses can assign their few highly skilled programmers to component construction and use less sophisticated developers to carry out the simpler assembly tasks. By making component standards available to the broadest possible spectrum of developers, DC Ps essentially drive those standards to market.

In this article, we review thestate of component softwareas embodied in DCPs. The two DCP market leaders are M icrosoft's DCOM (or ActiveX /DCOM ) and Sun's J avaBeans. H owever, Internet and $O$ bject $M$ anagement Group (OMG) component standards are emerging that will likely impact both the content and status of these two DCPs. We also discuss component framew orks, which extend DCPs to providemorecomplete application development solutions.

\section{DCP CONCEPTS}

Software components are reusable building blocks for constructing software systems. ${ }^{2}$ Components encapsulate semantically meaningful application or technical services, such as rating insurance applicants or authorizing client access to service resources. Components differ from other types of reusable software modules in that they can be modified at design time as binary executables; in contrast, libraries, subroutines, and so on must be modified as source code.

Component standards specify how to build and interconnect software components. They show how a component must present itself to the outside world, independent of its internal implementation. This singleminded emphasis on external interfaces and interaction protocols distinguishes component standards from other communication conventions. Well-thought-out component standards ensure that

- components with similar specifications are interchangeable and independently upgradable,

- developers can customize the appearance and behavior of components along predetermined dimensions, and

- components can be combined to form larger grained components as well as complete applications.

Thus, component standards play a critical role in ensuring that developers achieve the anticipated benefits from reusable components-enhanced productivity, uniformity, ease of use, and faster time to market.

\section{Component interfaces}

A component restricts access to its services and internal structures through one or more public interfaces. As Figure 1 shows, an interface defines a set of properties, methods, and events through which external entities can connect to, and communicate with, the 


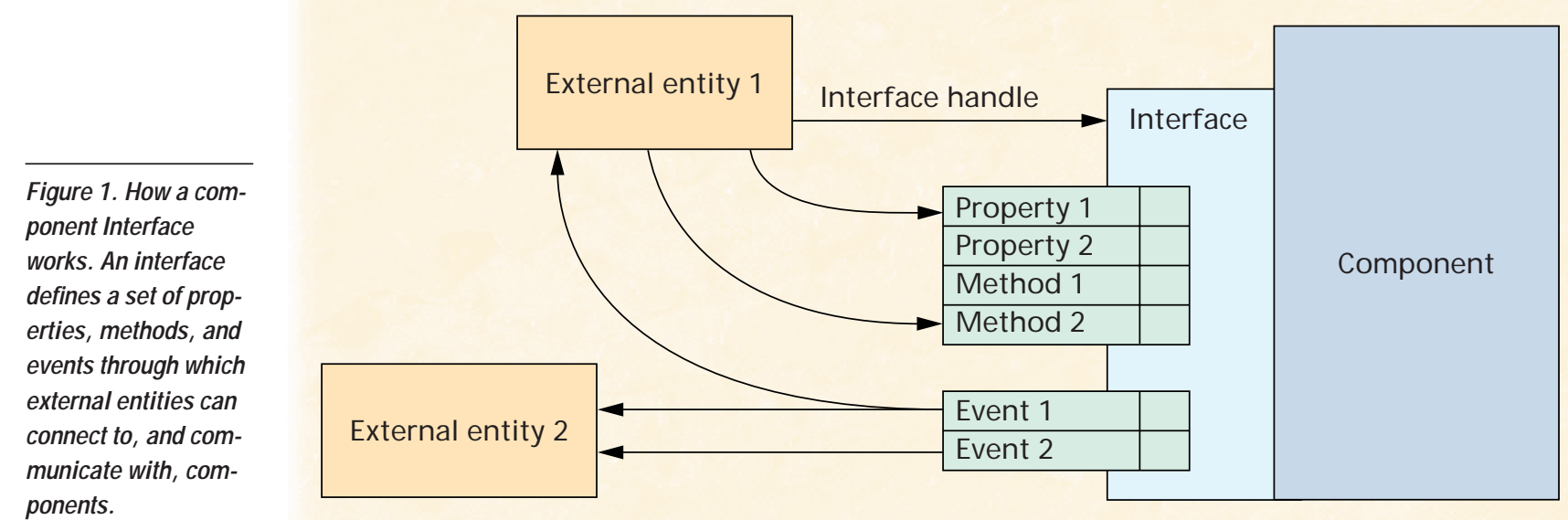

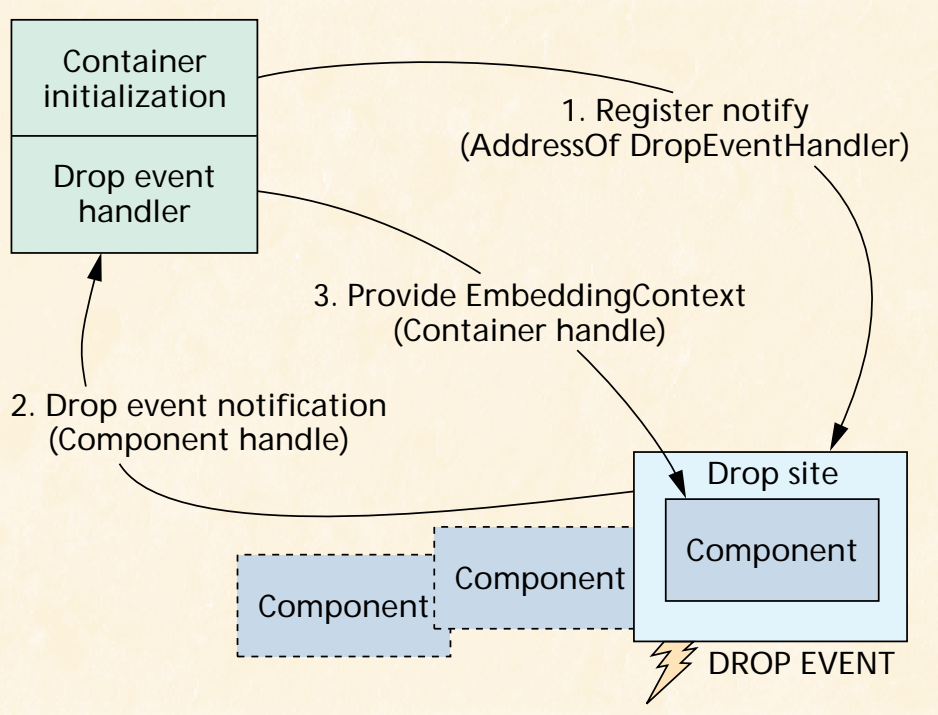

Figure 2. Event registration and notification. A drag-and-drop event is used to dynamically relate a newly embedded component to its container. component. Properties and methods typically represent a component's callable A PI-the protocol used by external entities to access a component's services. Properties expose a component's public attribute data via accessor operations; methods inaugurate a component's behavior. Events specify a component's response to external stimuli or internal conditions, such as a property value changing. The component interface specifies the signature of the event it will raise when the condition occurs. It does not know or care how that event is consumed or who the consumer is. Consuming entities are responsible for registering interest in the event and providing a handler for its occurrence. This publish-and-subscribemodel of interaction allows communication channels to be established dynamically.

\section{Containers}

Components exist and operate within containers, which provide a shared context for interaction with other components. Containers also offer common access to system-level services for a component's embedded components (such as process threads and memory resources). Containers are typically implemented as components, which can be nested in other containers. An example is embedding widget field arrays into panels within GUI windows. Event-based protocols are commonly used to establish the relationship between a component and its container.

Figure 2 illustrates dynamic event protocols for dragging and dropping a component onto a container. Upon initialization, the container registers its interest in drag-and-drop events with a drop site. (D rop sites are usually implemented as interfaces on the container itself.) Later, when a drop event occurs, the drop site notifies the container by calling the container's previously registered event handler, passing a handle to the dropped component. The event handler might change the appearance of its container's icon to, say, signal the user that the drag-and-drop operation has successfully completed. Typically, it will pass the dropped component a handle to the container, which lets the component access the container's environmental services.

\section{Pervasive metadata}

Component standards specify the self-descriptive information that a component must publish to flexibly communicate with other components. This metadata lets containers, scripts, development tools and other components discover a component's capabilities- either statically at design time, or dynamically at runtime.

Figure 3 illustrates the basic categories of metadata used in DCPs. DCPs generally implement metadata as a type of component whose interfaces are termed introspection or reflection. The italicized terms correspond to the categories in the figure.

- Component info describes the component's general compile-time and runtime properties, including where to find it and how to activate it (for example, a path name and its process or its caller process).

- External references point to metadata that describes other components.

- Type descriptor and Type form the fundamental metadata elements. 




Figure 3. Generic model of metadata categories. Metadata lets containers, scripts, development tools, and other components discover a component's capabilities at either design time or runtime.

- Interfaces describe public attributes, events, and methods.

- Classes describe the implementation of one or more interfaces. The metarelationships betw een classes, types, and interfaces differ across DCPs.

- Attributes, methods, and events characterize the classes and interfaces. All have associated metadata.

- Return types and parameters specify method inputs and outputs.

Recent advances in DC Ps have been fueled primarily by enriching component metadata and exploiting it aggressively. For example, metadata drives component composition and dynamic collaboration, enabling components that discover and manipulate each other's interfaces at runtime. Similarly, tools that extend development environments, such as object browsers, debuggers, and smart code editors rely on component metadata to populate themselves. A DCP's longevity will depend strongly on the expressiveness and extensibility of its metadata model.

\section{Integr ated development envir onments}

The notion of a component is inseparable from the notion of a component development environment or builder. The value of a DCP depends heavily on the efficacy and usability of its integrated development environment. IDEsare rapidly moving from text-based programming toward the direct manipulation of visually rendered components. Commercial IDEs include

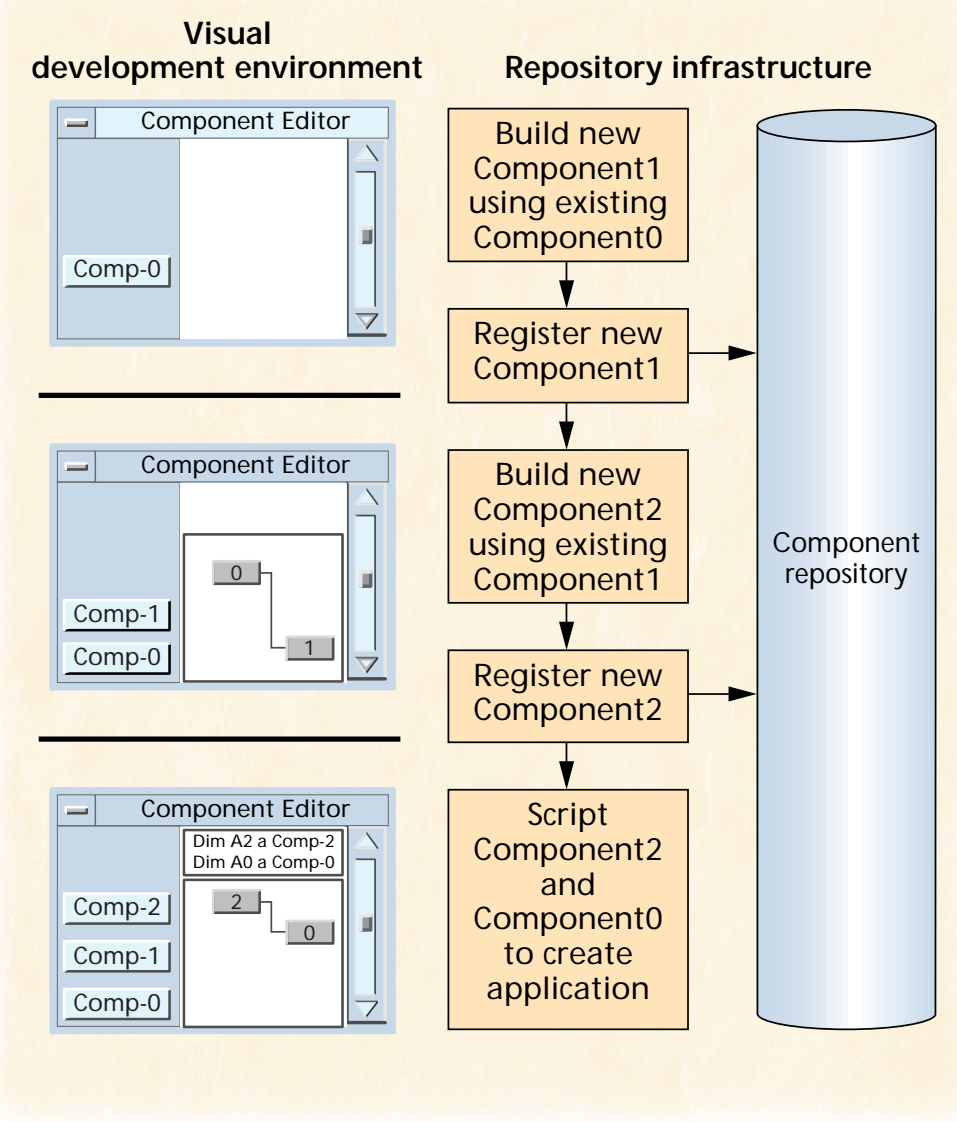

M icrosoft's Visual Studio, IBM 's VisualA ge for Java, and Symantec's VisualC afe, supplemented with scripting languages such as VBScript and JavaScript.

IDEs typically include or are evolving to include

- one or more palettes for displaying available components (rendered as icons);

- a "canvas" container onto which components are placed and interconnected, typically through drag-and-drop operations and pop-up menus;

- property and script editors that let users customize components within their containers;

- editors, browsers, interpreters, compilers, and source-level debuggers for developing new components and testing and refining component applications;

- a component repository and associated designtime browser services to locate components by matching user search criteria and using inspectors to view component metadata; and

- configuration management tools that structure and coordinate team-based development and release processes-tools that are essential for large software projects.

Figure 4 depicts a scenario in which a developer uses an IDE to construct an application by composing new components visually and scripting interactions between existing and new components.

This scenario illustrates that an IDE not only must
Figure 4. Using an integrated development environment (IDE) to build an application with existing and new components. The developer scripts component interactions. 


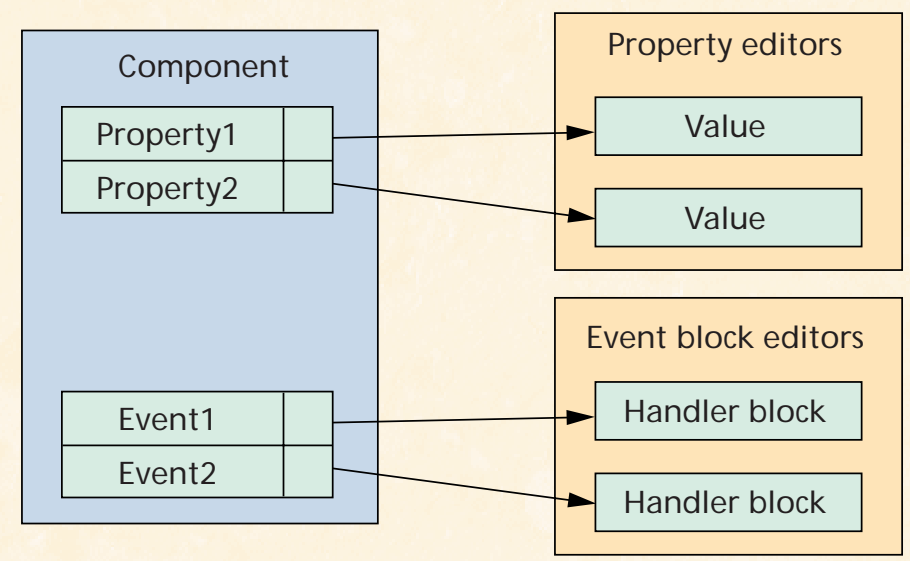

Figure 5. Customizing components in the IDE.

support the creation and composition of reusable components, it must also act as a framework for seamlessly integrating new components into itself. The IDE relies on the DCP's component standard to ensure agreement among the components, containers, and IDE on the patterns for hierarchical composition and metadata exchange.

D evelopers can also customize existing components by setting property values and providing new handlers for their events, as shown in Figure 5. Developers modify components either explicitly, via IDE menus and editors, or implicitly, as side effects of visual manipulations such as dropping component icons onto containers. Either way, the IDE must know how to access a component's metadata to display and populate editors or handle drag-and-drop events.

Components that can be customized must know if they are executing in a runtime or design-time context. Components expose different interfaces and display different behaviors at these times. During design, the component collaborates with the IDE through its metadata to expose its property and event editors. At runtime, however, the component manifests the behaviors specified during design. For example, clicking on a button component during design generally opens an event editor; at runtime, the same mouse click signals the button's window container to perform some action, such as closing itself.

\section{Distributed server components}

Enterprise computing relies on a robust set of services for accessing and managing shared services, information, and computing resources. To move beyond the desktop, components require five distributed services:

- Remote communication protocols, which enable interactions at the application layer among components distributed over a network. Protocols can be synchronous (for example, remote procedure calls) or asynchronous (for example, mes- saging services that enable an efficient, nonblocking store-and-forward model).

- Directory services, which provide a coherent global scheme for naming, organizing, and accessing shared services and resources.

- Security services, which protect shared resources by ensuring that communicating parties are properly authenticated and have suitable authorization, and that third parties have not intercepted or tampered with their messages.

- Transaction services, which coordinate concurrent updates to enterprise-critical data, and ensure that all updates leave data in correct and consistent states.

- System management services, which provide a unified set of facilities to monitor, manage, and administer services and resources across the enterprise.

A large installed base of mature products that support distributed-service APIs already exists. H owever, because these product sets deliver overlapping services through different APIs, it is difficult to sel ect products and integrate their services into applications. This makes it much more challenging to design enterprise server components that can effectively incorporate distributed services.

In fact, many distributed facilities are not services in the traditional client-server sense. Rather, they constitute part of a component's runtime environment. For example, a server component may obtain transactional persistence from its runtime context, rather than implementing it directly. The relationship between transaction context and server components is roughly analogous to visual containment of desktop components.

DCP providers are attempting to generalize containment models to encompass the relationships between server components and their runtime hosts; like visual containers, transaction contexts provide runtime access to basic life-support and custodial services for their components. H owever, server components generally provide concurrent services to many users, whereas desktop components support single users. Also, server components are often multithreaded, replicated, and pooled, to achieve scalability and reliability. Consequently server components can't readily be organized into static containment hierarchies. In short, the complexities of distributed computing threaten the viability of container-based models for designing and using server components.

\section{DCOM}

$M$ icrosoft's DCP is based on DCOM, which consists of the Component $\mathrm{O}$ bject $\mathrm{M}$ odel ( $\mathrm{CO} M$ ) binary standard, augmented with a runtime infrastructure to 
support component communication across distributed address spaces. ${ }^{3-4}$ (Earlier CO M -based incarnations were named VBX and OCX, for VisualBasic and OLE controls, respectively.) DCOM specifies the type and the structure of the interfaces components must implement. All DCOM components must implement at least one interface, IUnknown, which supports basic mechanisms for casting interface references and reference counting. . $^{5-7}$

DCO M itself is a relatively simple component standard; its utility resides more in specialized interfaces, such as compound documents, drag-and-drop, and persistent streaming and storage. DCO M 's event notification mechanism is implemented through IConnectionPoint and several related interfaces.

DCOM component interfaces are specified using an Interface D efinition Language (IDL) derived from the $O$ pen Software Foundation (OSF). DCOM is independent of language and implementation. Until recently, DCOM was restricted primarily to the Windows platform. M aturing ports to Unix and $M$ acO $S$ are reducing D CO M 's platform dependency.

\section{Development envir onment}

M icrosoft's Visual Basic 3.0 provided a rudimentary but productive environment for assembling VBX components, which have since been replaced with components based on the DCOM standard. In addition, M icrosoft has infused progressively more component-oriented programming capabilities into its IDEs. For example, VB 5.0 lets developers create new components as well as use existing ones. In addition, the VB IDE now creates much of the boilerplate code components require at runtime. For example, it automatically generates and registers component interfaces and metadata.

The M icrosoft IDE currently supports component development in three languages-Visual Basic, Visual $\mathrm{C}++$, and $\mathrm{J}++$ (M icrosoft's J ava). The IDE is itself an application built using the native DCO M model, making it extensible and customizable through standard DCOM mechanisms. It also provides mechanisms (add-ins) that help developers customize it to, say, have a different look and feel or enforce desired development patterns.

\section{Metadata}

The basic COM model supports a rudimentary form of component self-description through IUnknown. In addition, a set of metadata components, Type L ibraries, express in machine-readable form what IDL expresses in human-readable form.

A Type Library collects metadata for its associated component and provides ITypeLib and ITypeInfo interfaces to access and navigatethe metadata. A Type Library contains five general kinds of information:
- CoClass is a metadescriptor for a COM object, describing all incoming and outgoing interfaces for that COM class, including public properties and methods.

- Interface provides memory layout and descriptive information for public operations such as names, return-type, and optional dispatch identifier.

- Module describes the dynamic linking library (DLL) module, including path name global variables and exported functions.

- Typedef is the metadescriptor for userdefined data structures.

- Importlib provides a way to get the metadescriptor for a referenced Type Library.

Each metadescriptor specifies a common set of properties, including a human-readable name, machine-readable globally unique identifier (GUID), version, documentation string, help file name, and flags (for example, hidden or restricted).

Given a class identifier (CLSID), a client can get a handle to a component's Type Library and query its metadata without an instance of the component. Alternatively, if a running component instance is available, a client can obtain the Type Library through the instance's IProvideClass Info interface. The first modetypifies a design scenario. The second modeaids in dynamically discovering interfaces and in component collaboration at runtime.

\section{Server platform}

$M$ icrosoft is strongly committed to delivering a credible server component platform by successively enhancing the N T operating system. N T's current and beta-level distributed enterprise services include

- Remote communication protocol. DCOM uses remote procedure calls to communicate across the network. M icrosoft's R PC, derived from the OSF DCE RPC, allows communication among distributed components through a standard proxy/stub mechanism. M icrosoft also plans to support an asynchronous protocol directly in DCOM , most likely through tighter integration of their messaging product, M S M essage Q ueue.

- Directory services. M icrosoft Active Directory combines D omain $\mathrm{N}$ aming System, the dominant Internet name resolution scheme, with the Lightweight Directory Access Protocol (LDAP), which is ISO X.500 compliant. M icrosoft plans to integrate these services into the next release of Windows N T.

- Security services. M icrosoft N T provides Secure Sockets Layer public-key-based security and a proprietary security protocol that is based on NT
DCP providers

are attempting

to generalize

containment models

to encompass the

relationships

between server

components and

their runtime hosts.

\author{
(n)
}

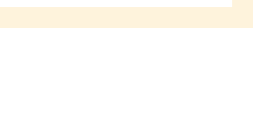


DCOM is languageneutral but platform-dependent; JavaBeans is platform-neutral but languagedependent.
LAN M anager (NTLM). H owever, the next release of NT will include a security service compliant with Kerberos 5.0 and tightly integrated with M icrosoft's A ctive Directory.

- System management services. NT 4.0 includes a $\mathrm{M}$ anagement Console ( $\mathrm{M} \mathrm{M} \mathrm{C}$ ) that comprises a UI container for third-party systems management components. NT 5.0 is slated to provide a true management information bus that will support mechanisms to monitor and manage the resources and services on a distributed NT installation. Thebus will also publish a set of A PIs for integrating existing system management products.

- Transaction services. The M icrosoft Transaction Server integrates transaction services into the component development model and provides a transactional runtime environment for server components. M TS defines ObjectContext, an analog to containers for server components. Server components access their operational context through this component's IObjectContext interface. Components created within, or added to, an ObjectContext participate transparently in that context's transactions and share that context's associated security.

\section{JAVABEANS}

J avaBeans has quickly gained market attention, emerging as the dominant competitor to DCOM . Whereas DCOM is language-neutral but platformdependent, JavaBeans is platform-neutral but language-dependent. DCO M capabilities are built up by composing more el ementary binary components. For example, the IPersist interface is composed from IS torage and IStream. In contrast, JavaBeans component capabilities are implemented as a set of language extensions to the standard J ava class library. Thus, J avaBeans is a set of special ized J ava programming language interfaces. It achieves platform portability through the J ava Virtual $M$ achine.

Like DCO M, J avaBeans interfaces expose properties, methods, and events. The J avaBeans property model is richer than DCOM 's: In addition to singleand multivalue properties, JavaBeans defines bound and constrained property types. Bound properties use Java events to notify other components of a property value change; constrained properties let those components veto a change. Constrained properties provide a uniform language-based approach to basic validation business rules. Both bound and constrained properties are missing from most object-based systems. They let developers factor application logic in a modular and maintainable way that makes it easy to preserve the consistency of business data.
JavaBeans' event notification mechanism involves three related Java-level class interfaces-Event, EventSource, and EventListener. EventSource notifies all registered EventListeners, passing each an Event object when the event of interest occurs. The event model supports two other features that enhance ease of use. Event Sources default to multicast but can beset to unicast-allowing at most one EventListener. EventAdapters can be added to relieve developers from having to write handlers for all the events defined in a listener's interface.

In addition to component interface constructs, $J$ avaBeans incorporates mechanisms for component containment and pervasive metadata analogous to DCOM. H owever, DCOM components implement persistent identity through GUIDs. J avaBeans use string names, which may not be globally unique.

\section{Development envir onment}

TheJ avaBeans A PI explicitly supports visual development of Bean components using property sheetsbuilt-in property editors and editor aggregations. This IDE or application builder support is analogous to DCOM 's IPropertyPage interface.

A growing number of IDEs (including Symantec's VisualC afe, IBM 's Visual Age for Java, Borland's JBuilder, and Sun's J ava Workshop) support visual development with property sheets, palettes, and design-time drag-and-drop behaviors. These tools offer productivity features comparable to the $M$ icrosoft IDE. In addition, their visual model for interconnecting components is significantly more expressive and intuitive than the current versions of the M icrosoft IDE.

\section{Metadata}

J avaBeans inherits Java's Core R eflection API, which provides a specialized set of classes for metadata. Each of J ava's methods, fields, constructors, interfaces, and classes has a corresponding metadata class that supports dynamic interrogation, instantiation, and invocation. Unlikethe J ava language, A N SI C + and Visual Basic don't have built-in reflection support. Consequently all metadata on DCOM (except for $\mathrm{J}++$ ) is in the component model, not the language.

In addition to J ava's Core R eflection API, J avaBeans provides an Introspection interface that returnsa different set of metadata classes tailored to support component-based development. For example, the visual icon that a Bean displays on an IDE's palette is specified by the Bean Info metadata class. The other JavaBeans metadata classes are derived from a common base class, FeatureDescriptor, and roughly correspond to DCOM metadata, ${ }^{8}$ as summarized in Table 1. To foster the systematic use of JavaBeans metadata, the DCP provides a utility class, 
Table 1. Comparison of DCOM and JavaBeans metadata.

Generic metadata
Cass
Type
To Get from running object
To dynamically instantiate a class
To get reflected member
information

To navigate up

To dynamically invoke a method

ComponentInfo

External References

Standard metadata conventions
DCOM

CoClass, ITypelnfo

TypeDef

AnObject.IprovideClassInfo()

ITypelnfo. Oreatelnstance()

GetFuncDesc(), GetTypeAttr(),

GetVarDesc(),GetNames()....

ITypelnfo.GetContainingTypeLib()

Invoke()

ITypeLib.GetLibAttr()

ITypelnfo,GetDLLEntry()

ImportLib

Eement Attributes
Java Core Reflection

Class

Class

anObject.GetClass()

aClass.newlnstance()

GetClasses(), GetFields(), GetEventDescriptors,

getMethods(),

getConstructor()

GetDeclaringClass()

Invoke()

Package import conventions
GetMethodDescriptors,

GetPropertyDescriptors

BeanDescriptor, Beanlnfo

JavaBean, Beanlnfo

BeanDescriptor.GetBeanClass

FeatureDescriptor
Inspector, which navigates the Introspection and Core Reflection APIs.

Despite their many useful features, the JavaBeans metadata facilities rely heavily on error-prone naming conventions called design patterns. For example, the accessor and mutator methods for a property $X$ must be named GetX and SetX in order for the Introspection interfaces to work properly. EventListeners and Bean Info names are similarly constrained by string-oriented templates.

\section{Distr ibuted server components}

Sun has recently released a preliminary specification for Enterprise JavaBeans (EJB). The goal is to provide the same kind of scalable, enterprise-capable server environment for JavaBeans that M icrosoft is delivering and hardening for W indows N T servers. ${ }^{9}$ The standard explicitly specifies that all Enterprise J avaBeans will run inside a container that isolates the J avaBean from the server execution environment. The container automatically allocates process threads to the component and manages concurrency, security, persistence, and transactional activities on behalf of the component. EJB's server environment includes

- R emote communication protocol. JavaBeans has full access to Java's native remote method invocation (RMI), which runs directly on top of TCP/IP. However, enabling RMI for a class requires making explicit changes to an existing Java class. Also, instances of remote classes cannot be passed by value.

- D irectory services. Sun has released a beta version of the Java N aming \& D irectory Interface (JNDI), which provides an implementationindependent API that allows applications written in J ava to leverage existing directory services such as LDAP and Domain N ame System. EJB containers must be locatable through the J N DI.

- Security services. EJ B can use all security services specified by the standard java.security package. This includes public- and private-key authentication, encryption, digital key manage ment, and access control lists.

- Systems management services. At the time of this writing, Sun has not met its scheduled public released date of fourth quarter 1997 for the 1.0 specification for its Java $\mathrm{M}$ anagement $\mathrm{API}$ (J M API). H owever, available documentation indicates that J M API will specify a comprehensive set of monitoring, management, and administrative services, including a UI style guide for an administrator's console (http://java.sun.com/ products/J avaM anagement/document.html).

- Transaction services. The EJB specifies a flat transaction model based on the $\mathrm{OM} \mathrm{G}$ 's $\mathrm{O}$ bject Transaction Service, explicitly discouraging use of the existing Java Transaction Service (JTS) API. Instead it delegates transaction management to the Bean's container.

J avaBeans components can be packaged for embedding in containers that support M icrosoft's D CO M component model, including Visual Basic, Internet Explorer, $\mathrm{O}$ ffice, and L otus $\mathrm{N}$ otes. This form of interoperability is driven by a communication bridge. The core technology behind the bridge is a packaging utility that generates an OLE Type Library and W in32 registry information for selected JavaBeans. The resulting data lets DCOM containers properly analyze, present, and manipulate Beans (for example, catching Bean events, invoking Bean service methods, and creating property sheets to customize Beans).

\section{DISTRIBUTED COM PONENTS ON THE WEB}

The Internet and private intranets are increasingly 


W3C efforts align
with DCPs in
assuming that
pervasive metadata
enables
semantically rich
interoperability,
whether between
components or Web
documents.

perceived as critical to enterprise computing architectures. Unfortunately, attempts to use the Web as a platform for distributing component applications have been impeded by the limited expressiveness of the HTM $L$ document standard.

As a short-term response, the World Wide Web Consortium (W3C) has incorporated an 〈object> tag into HTM L 4.0. W3C is also sponsoring more broadly based architectural solutions. These emerging standards could potentially lead to a convergence between component and Web document architectures.

Extensible $M$ arkup Language, or XM L (http://www.w3.org/TR/REC-html40/), is a metamodel for structured document exchange based on the Standard General M arkup L anguage (SG M L, an ISO standard). HTM L restricts Web documents to a predefined set of tags for specifying content and format. In contrast, X M L supports the definition of customized markup languages. For example, XM L tags could be defined for classifying component applets according to company- or industryspecific classifications.

The Resource Description Framework, or RDF (http://www.w3.org/T R/W D-rdf-syntax) is a metamodel, to be expressed using $X M L$, for capturing metadata about Web resources. Such metadata could be used by search engines, automated agents, and other Web client and server applications for component searching, rating, access control, licensing, and management.

The Document Object Model, or DOM (http://www.w3.org/TR/WD-D OM ), specifies automation interfaces through which scripts or applications can access and manipulateWeb documents. Given suitable XM L tags, DOM would allow Web documents to be manipulated as components or containers.

These W 3 C specifications represent a coordinated attempt to define object semantics for lightweight networks of distributed documents. They appear to accommodate component-level semantics as well. W $3 C$ efforts align with DCPs in assuming that pervasive metadata enables semantically rich interoperability, whether between components or Web documents.

\section{OMG COM PONENT STANDARDS}

The $O$ bject $M$ anagement $G$ roup has played a leading role in establishing open system specifications for distributed object computing..$^{10}$ Until recently, OM G focused on object-level standards. R esponding to easeof-use concerns from members aligned with the J avaBeans standard, the OM G issued a request for proposal for component-level specifications last year (http://www.omg.org). The RFP identified four core categories of requirements for standards:
- a component model that defines a component type system; interfaces for exposing and managing properties; mechanisms for raising and handling events; life cycle structure; and serialization;

- a component description facility that consists of a reflective information model supported by existing or new CORBA-related repositories;

- a programming model that maps component descriptions to languages that support CORBA IDL mappings, and that lets components be passed as value parameters in CO RBA requests; and

- a mapping to the JavaB eans component model that supports both design and runtime interoperability needs, including component inspection and the automated generation of software to integrate CORBA components into Java-based tools.

The OM G has several other RFPs in process for standards relating to the component model category. O ne RFP addresses the problems of composing objects using multiple IDL interfaces (for disjoint services) and of resolving conflicts among multipleinterfaces on a given object. A second RFP solicits proposals for interfaces to pass CO RBA objects by value parameters in CORBA object operations. Currently, CORBA supports the passing of object parameters only by reference, which impedes the port of RM I from a J ava-based protocol to IIO P. A third RFP focuses on scripting languages to support automation for CORBA components.

O M G generates specifications rather than software products or DCPs. Some DCPs, such as J avaBeans, are virtually certain to comply with these standards, while others such as D COM (ActiveX) probably will not. H owever, the O M G developed dedicated specifications to ensure interoperability of CORBA and COM objects to reflect M icrosoft's dominance of desktop computing. Similar market pressures will likely lead to analogous OM G specifications for the interoperability of DCOM and CORBA components.

\section{COM PONENT FRAMEWORKS}

DCPs do not of themselves guarantee complete and satisfactory software applications. They help users construct, reuse, and connect components, but they supply no guidance for application-level semantics or structure-how to design and arrange specific components to solve specific business problems. They also don't guarantee robust, scalable, and agile systemsareas that continue to require considerable engineering experience and discipline.

Component frameworks are an increasingly popular strategy for augmenting DCPs to fill these gaps. A component framework is a concrete implementation of one or more design patterns tailored to a particular business or technical domain, such as help desks, health care, or user interface construction. ${ }^{11} \mathrm{~A}$ design pattern 


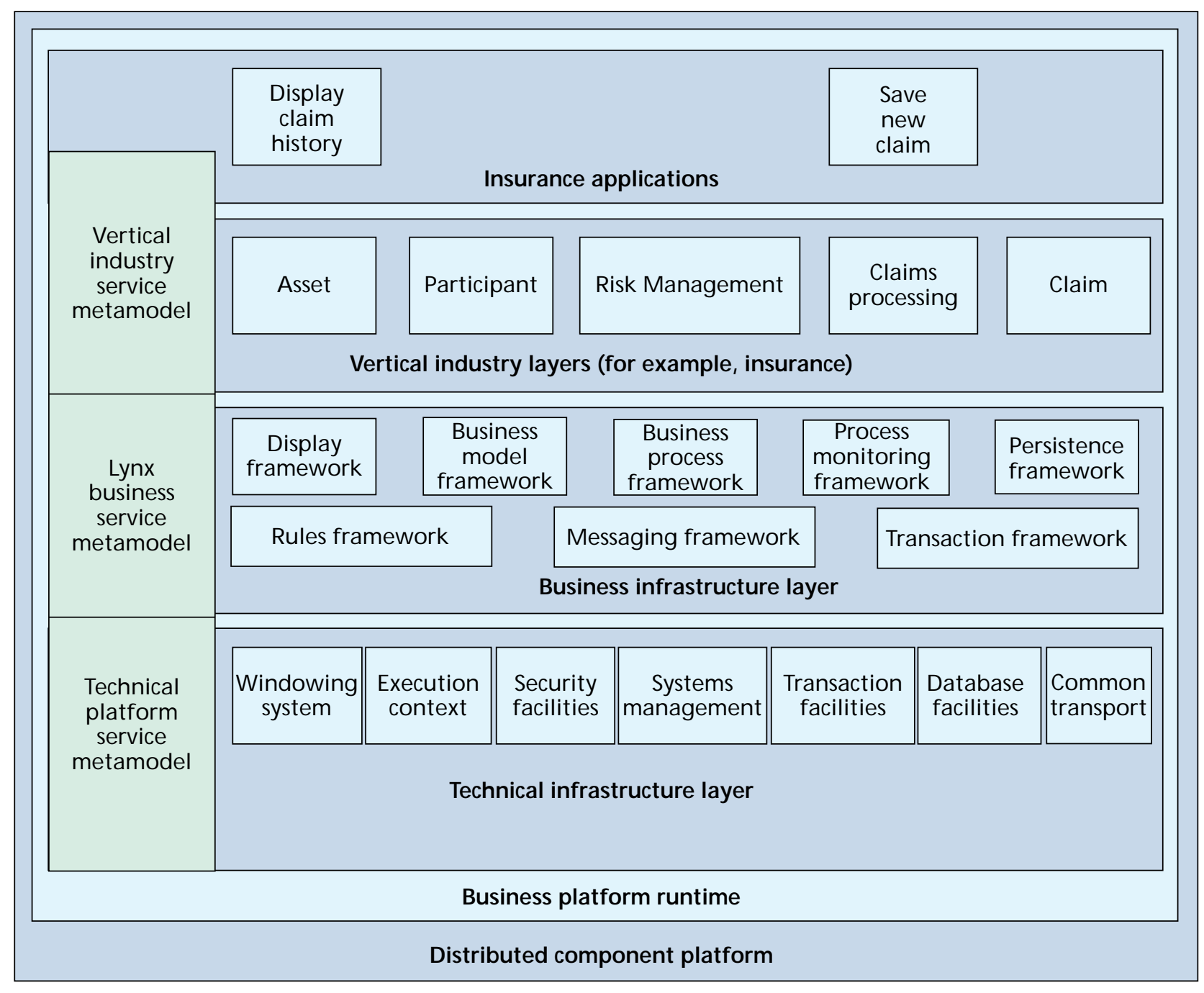

Figure 6. The Lynx framework extends directly out of the DCP substrate. Lynx's prefabricated components are organized into layers that expose a succession of technical and business services. Each layer is segmented into functionally specialized frameworks. At the lowest layer, Lynx simply packaged technical service APIs to achieve high performance and scalability. Each higher layer infuses a more specialized business perspective that hides the complexities of the lower layers. All layers conform to the DCP's uniform programming model.

expresses an abstract solution to a recurring design problem, as a set of components and component interactions. ${ }^{12}$ Examples includetheSmalltalk M odel-View Controller and market auction patterns, which help synchronize graphic displays and allocate scarce resources, respectively. A framework methodology guides application developers in determining exactly what to build and how to build it using that framework.

Computer Sciences Corp.'s Lynx framework illustrates how a component framew ork can extend a DCP into a complete development solution. Lynx provides a prebuilt application skeleton of component templates tied together by collaborative patterns. D evelopers extend the templates with business-specific components to construct custom applications such as insurance underwriting, toll collection, process control for plastics manufacture, and customer service.

As do all component framew orks, Lynx defines and factors functionality into components and patterns on the basis of specific technical and business design goals. CSC's focus is on large-scale, mission-critical business systems, so Lynx's overarching goal is to support high-volume, onlinetransaction-processing applications. Typical Lynx applications support thousands of users, performing 20 to 30 complex business work units per second with subsecond response times. (We use "business work unit" instead of "transaction" to avoid confusion with TPC database transaction metrics. Business work units span multiple tiers, and may encompass a few or dozens of TPC transactions, depending on the application.) Lynx aims to balance performance and scalability against secondary goals of developer productivity and application agility; Lynx lets developers adjust this mix systematically by customizing or extending framew ork components and patterns to reflect application-specific trade-offs.

A sFigure 6 shows, Lynx is actually a framework of frameworks, which collaborate to achieve a set of goals. Lynx attacks its performance goal by minimizing the number of process boundary traversals 
The use of DCPs for large projects will hinge on the availability of robust component-oriented methodologies. required to complete business work units: The transaction and message-routing framew orks collaborate to package an entire work unit into a single message. Lynx addresses scalability by minimizing the scope and duration of locks against shared services. For example, the messaging framew ork uses remote service proxies with connectionless service references rather than R PC-style proxy stubs; overall concurrency is maximized by preventing any one client from monopolizing access to shared middle-tier services. M essage routing and system monitoring also cooperate for scalability, which allows dynamic replication and distributed load balancing of middle- and back-end services to accommodate increased demand.

Inappropriate coupling between components makes applications less flexible. Lynx minimizes design-time dependencies betw een components that capture the core business model and those that support presentation, workflow, and database persistence. Isolating components by functional roles means that part of an application can change with little or no effect on the other parts, promoting agility. For example, persistence implementations can be tuned or switched without affecting business or presentation components. Isolation also fosters productivity because developers can train quickly and specialize on manageable pieces of the framew ork.

Lynx's layering scheme isolates application-level development from the complexities of the technical infrastructure, which also contributes to agility. To date, we have implemented Lynx on two distinct platforms with minimal architectural and design changes: Forte Software's distributed object development environment and M icrosoft's D CO M. Lynx's higher layers preserve and expose both patterns and metadata from low er layers, allowing selective adaptation and tuning. Finally, layering promotes extensibility, both vertically and horizontally. For example, weare incorporating frameworks for finance, process control, legacy integration, business rules, and electronic commerce. In contrast to J avaBeans' constrained properties, the rules framew ork supports rules that connect multiple attributes across business entities (for example, to constrain the salaries of managers and their employees).

Tightly coupled to the Lynx framew ork is a methodology that defines the processes, techniques, work products, and management model for using Lynx productively. The methodology adapts and unifies a set of standard analysis methods for static and dynamic object modeling. These methods are synthesized with techniques for modeling and reengineering business processes. The methodology then specifies how to map the resulting business object and process models onto the Lynx framew ork in terms of components, business services, application tasks, and GUI storyboard layouts. For example, business entities, such as Policy or Claim are translated into sets of collaborating display, business, and persistence components. Because the default components and collaboration patterns are uniform across business components, Lynx can exploit automated code-generation techniques at the framew ork level to maximize productivity. Project management processes are driven by a road map that defines development activities, their iteration and sequencing, dataflows, work roles, and team structures.

Frameworks such as Lynx embody architectural blueprints for building component-based applications. Framew orks and their supporting methodologies augment DCPs to minimize risk and help ensure design uniformity and semantic interoperability across applications.

omponent software standards continue to evolve, along a fault line formed by rival technology sets from M icrosoft and Sun. The distributed component platforms that extend and package these standards are maturing rapidly into commercial products accessible to a broad spectrum of developers.

Interest in component software has grown and intensified in recent years. Business interest has been driven by competitive pressures to deliver agile applications more rapidly and economically. Developer interest derives from the ease of use of the latest IDEs, which approaches the satisfying tactile experience obtained from assembling physical parts. Finally, technical interest has been driven by three converging trends for reuse. The patterns movement fosters reuse at the level of software designs. Component frameworks promote reuse of design patterns and code. Component ID Es enablethe construction and deployment of new frameworks, driving reuse of entire application skeletons.

Important challenges remain for component software in enterprise settings. DCPs continue to expose technical complexity to users: Server components and supporting IDE extensions for developing, using, and managing them are in their infancy. Standards for deploying components across intranets to Web clients aresimilarly immature. In addition, current DCPs support only basic one-to-one interactions between remote components. Such substrates provide meager support for developing the complex coordination patterns required for collaborative workgroup applications, such as voting, negotiation, or sharing.

The use of DCPs for large projects will hinge on the availability of robust component-oriented methodologies. 
The value of DCPs to end users depends directly on a critical mass of ready-to-use components. This presupposes a healthy market for third-party components, complete with distribution channels, sustainable pricing models, and standards for packaging and certification. M arket growth also depends on some form of stabilization or resolution to the conflict between competing DCPs, most probably through utilities such as the JavaBeans/D COM bridge. Absent interoperability solutions and standards equilibrium, it will be difficult for component markets to expand.

To date, the majority of off-the-shelf components consist of business-neutral GUI controls, such as spreadsheets and graph or report generators. Components are needed that represent business-level entities and processes. Software vendors and industry consortia such as IBM , M icrosoft, and the OM G are actively pursuing generic business components targeted for various vertical markets. Prompt convergence on standards is critical to preventing the proliferation of custom components with incompatible business semantics. Such a trend would obstruct interoperability, reusability, and the growth of component markets.

We believe the ultimate value of software components lies in frameworks that infuse progressively more targeted business-level perspectives into DCPs. The goal is to replace IDE palettes of text fields, data grids, push-buttons, and similar GUI controls with palettes that contain business objects, services, and functional views. Developers would then select, customize, and assemble these items into specialized components such as insurance coverage, and script complex business processes such as order fulfillment. Thus, the real challenge of component software is to let devel opers build business systems on business component platforms, rather than software systems on software component platforms.

\section{References}

1. R. Adler, "Emerging Standards for Component Software," Computer, Vol. 28, N o. 3, M ar. 1995, pp. 6877.

2. A. Thomas, "A Comparison of Component M odels," Distributed O bject Computing, July 1997, pp. 55-57.

3. K. Brockschmidt, Inside O LE, 2nd ed., M icrosoft Press, Redmond, Wash., 1995.

4. N. Brown and C. Kindel, Distributed Component O bject M odel Protocol-D CO M /1.0, M icrosoft Corp.; Redmond, Wash., 1996, http:/www.microsoft.com/ oledev/olecom/draft-brown-dcom-v1-spec-01.txt.

5. DCOM Technical O verview, M icrosoft Corp., Redmond, Wash., 1996.

6. D. Chappell, ActiveX and O LE, M icrosoft Press, Redmond, Wash., 1996.
7. "DCOM Architecture," white paper, M icrosoft Corp., Redmond, Wash., 1997.

8. JavaBeans API Specification, Version 1.01, Sun M icrosystems, M ountain View, Calif., 1997.

9. A. Thomas, "Enterprise JavaBeans Server Component M odel for Java," prepared for Sun M icrosystems by Patricia Seybold Group, 1997; http://java.sun.com/ products/ejb/white_paper.html.

10. J. Siegel, CORBA Fundamentals and Programming, John Wiley \& Sons, N ew York, 1996. Current O M G specifications can be found on http://www.omg.org.

11. R. Johnson, "Framew orks = (Components + Patterns)," Comm. ACM , Oct. 1997, pp. 39-42.

12. E. Gamma et al., D esign Patterns: Elements of Reusable Software, Addison-Wesley, R eading, M ass., 1996.

David Krieger is the head of development and chief architect for the Lynx framew ork at CSC Consulting in Waltham, M assachusetts. Previously he worked in product development as a consulting engineer at L otus D evelopment Corp. and as a manager and technical architect at Bachman Information Systems. $\mathrm{H}$ is computing interests include large-system architectural patterns, distributed-object framew orks, and highvolume transaction processing. Krieger holds six patents in software modeling and visual development environments. He received a BS in computer engineering from Clemson U niversity and is a member of the IEEE Computer Society.

Richard M. Adler is an architect for the Lynx framework at CSC Consulting in Waltham, M assachusetts. Previously, he directed development of distributedcomputing tools at a middleware start-up company and consulted with N ASA on intelligent systems for launch support operations. $\mathrm{H}$ is computing interests include distributed and intelligent systems, software agents, and knowledge management. A dler received a BS and an M S in physics from the University of M ichigan and the U niversity of Illinois at U rbana, respectively, and a PhD in the philosophy of physics from the $\mathrm{U}$ niversity of $\mathrm{M}$ innesota. $\mathrm{H}$ e is a member of the IEEE Computer Society and the ACM .

Contact the authors at CSC Consulting, 266 Second Ave., Waltham, MA 02154; ddavid_krieger, richard_adler\}@csc.com. 